\title{
Green's Function Application to Photonic Crystal Waveguides Analysis
}

\author{
Yuriy E. Mitelman ${ }^{1}$, Sergey N. Shabunin ${ }^{1, a)}$ \\ ${ }^{1}$ Yeltsin Ural Federal University, Yekaterinburg, Russia \\ a)Corresponding author: shab@ieee.org
}

\begin{abstract}
The paper considers application of the Green's tensor functions to analysis of the multilayer magneto-dielectric structures in relation to photonic crystals of the microwave range. The solution of the problem of electromagnetic wave propagation in rectangular and flat waveguides, as well as in unlimited space is presented. Efficiency of application of the Green's functions to calculation of transmission coefficients through the regular and perturbed structure of a photonic crystal is shown. It was proposed to solve the problem of diffraction of electromagnetic waves by the layered structures as the problem of equivalent electric and magnetic currents radiation. These currents are determined on the illuminated layered media surface by the Equivalence Theorem. The magnitude of the current is related to the electric field intensity, and, also, the direction of the incident wave is defined by the current phase distribution. The radiation of the equivalent currents behind the layered structure is considered as a transmitted wave. Radiation of these currents in the same region is treated as a reflected wave. So, transmission and reflection coefficients are defined. Application of this approach to analysis of the periodic structures of the photonic crystals is shown in this paper.
\end{abstract}

Keywords: photonic crystal, propagation, transmitting losses, Green's function, diffraction.

\section{INTRODUCTION}

Structures with periodically changing electrical characteristics are often used as component parts of microwave devices. The similar structures, but with variable refractive indices, are used to create devices operating in the optical frequency band. Due to the periodic nature of changes in parameters along one, two or three axes of the coordinate systems, the considered structures are called the photonic crystals [1] - [3]. Currently, there are many publications on use of the periodic structures for the development of devices in the microwave, infrared, and optical frequency bands [4] - [10]. To calculate the diffraction properties of the photonic crystals, numerical methods are mainly used, for example, the finite element method. The software such as the CST Microwave Studio, ANSYS HFSS, and EMSS FEKO based on numerical methods is applied to modeling the electromagnetic waves propagation through these structures. At the same time, some special cases can be analyzed much faster using the analytical electromagnetics methods. One of the well-established methods that are often used for solving propagation, radiation, and diffraction problems is the Green's function method [11].

Application of the Green's functions method to design the multilayer radio-transparent radomes for antenna systems was proposed in [12]. It was shown by this method that the layered structures can be efficiently designed to transmit electromagnetic radiation of antennas with minimal losses through the antenna radomes in the given frequency ranges. In some cases, the multiband antenna radomes are successfully developed.

\section{ELECTRIC FIELD IN 1-D LAYER STRUCTURE}

Periodic structures with the rectangular and circular outer conducting surface, as well as the parallel-plate waveguides, can be considered as a cross-section of the one-dimensional photonic crystal. In the infrared and optical

International Conference of Numerical Analysis and Applied Mathematics ICNAAM 2019

AIP Conf. Proc. 2293, 140015-1-140015-4; https://doi.org/10.1063/5.0026827

Published by AIP Publishing. 978-0-7354-4025-8/\$30.00 
frequency bands, the finite and infinite rectangular and circular dielectric waveguides are used. In the 1-D photonic crystals, refraction indexes, dielectric and magnetic permeability alternate according to a certain law (Fig. 1). To consider electromagnetic losses in the crystal material, its conductivity is taken into account. Losses in the conductive waveguide walls are considered by introducing a non-zero surface impedance of the wall material. The frequency dependence of the transmission coefficient changes dramatically when a layer with other electrical parameters or thickness appears in the structure (the perturbation occurs).

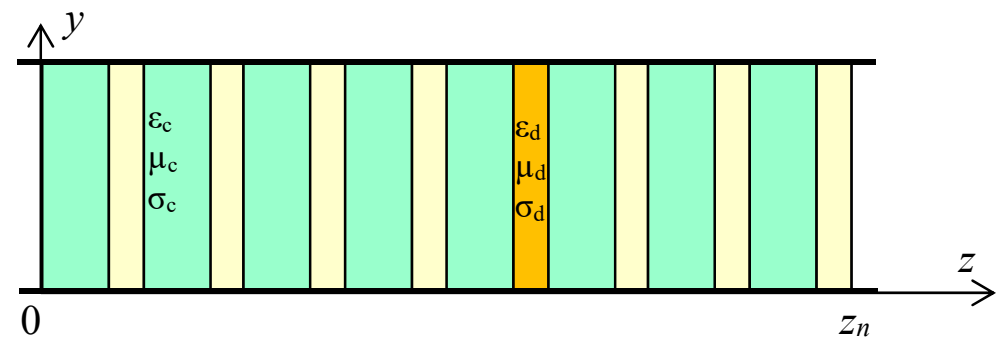

FIGURE 1. 1-D photonic crystals structure with the disturbed layer.

As the first approximation, the transmission coefficient of the electromagnetic wave through the periodic structure can be determined from the solution of the problem of the Hertzian dipole radiation from the outer side of the photonic crystal. The electric field at an arbitrary point in the space given by the vector $\mathbf{r}$ is defined as follows:

$$
\mathbf{E}(\mathbf{r})=\int_{S^{\prime}} \overline{\bar{\Gamma}}_{11}\left(\mathbf{r}, \mathbf{r}^{\prime}\right) \mathbf{J}\left(\mathbf{r}^{\prime}\right) d s^{\prime},
$$

where $\overline{\bar{\Gamma}}_{11}\left(\mathbf{r}, \mathbf{r}^{\prime}\right)$ is the electric Green's function. Let the electric dipole is oriented along the $y$-axis, so

$$
\mathbf{J}\left(\mathbf{r}^{\prime}\right)=\vec{y}_{0} I_{0} \delta\left(x^{\prime}-x_{0}\right) \delta\left(z^{\prime}-z_{0}\right) .
$$

The apostrophe denotes the coordinates of the source point.

The electric field excited by the dipole has three components. However, for the given length of the periodic structure under discussion, the contribution of the longitudinal component will be negligible. Taking into account the polarization characteristics of the electromagnetic field, the $E_{x}$ and $E_{y}$ components are of interest for the electromagnetic wave propagating along the $z$-axis. For example, the $E_{y}$ component is calculated using the $\Gamma_{11 ; y y^{\prime}}$ component of the Green's function. This component in the Cartesian coordinate system is written as follows:

$$
\Gamma_{11 ; y y^{\prime}}\left(\mathbf{r}, \mathbf{r}^{\prime}\right)=\sum_{m, n}\left[\left(k, k_{m n}^{e}\right)^{-2} \frac{\partial}{\partial y} \chi_{m n}(\rho) \frac{\partial}{\partial y^{\prime}} \chi_{m n}^{*}\left(\rho^{\prime}\right) \frac{\partial^{2} g\left(z, z^{\prime}\right)}{\partial z \partial z^{\prime}}+\left(k_{m n}^{h}\right)^{-2} \frac{\partial}{\partial x} \psi_{m n}(\rho) \frac{\partial}{\partial x^{\prime}} \psi_{m n}^{*}\left(\rho^{\prime}\right) f\left(z, z^{\prime}\right)\right]
$$

where $\chi_{m n}(\rho)$ and $\psi_{m n}(\rho)$ are the electric and magnetic eigenfunction for the selected cross-sectional area; $k_{m n}^{e}$, $k_{m n}^{h}$ are the proper eigenvalue, $\rho=(x, y)$. The asterisk indicates the conjugate function. The summation sign in (3) denotes summation for a finite area and integration for an infinite area.

We assume that in the plane transverse to the $z$-axis the medium is homogeneous. The model of an equivalent circuit consisting of two lines [13] of the electric and magnetic type is used. Since the source and the point of view are at different regions, the transmission coefficients $T_{i}^{E, M}$ of the $i$-th layer in the electric and magnetic equivalent circuits were added:

$$
T_{i}^{E}=\frac{Y_{i}^{E}}{Y_{i}^{E} \cos \gamma_{i i}^{E} d_{i}+j Y^{E}\left(z_{i}\right) \sin \gamma_{i}^{E} d_{i}}, T_{i}^{M}=\frac{Y_{i}^{M}}{Y_{i}^{M} \cos \gamma_{i}^{M} d_{i}+j Y^{M}\left(z_{i}\right) \sin \gamma_{i}^{M} d_{i}} .
$$


If the photonic crystal in the rectangular waveguide is under consideration as shown in Fig. 1, the Green's function component $\Gamma_{11 ; y y^{\prime}}\left(\mathbf{r}, \mathbf{r}^{\prime}\right)$ is described as follows:

$$
\Gamma_{11 ; y y^{\prime}}\left(\mathbf{r}, \mathbf{r}^{\prime}\right)=\sum_{m=0}^{\infty} \sum_{n=0}^{\infty} \frac{1}{a b} \frac{\frac{\varepsilon_{m} \varepsilon_{n}}{k^{2}}\left(\frac{n \pi}{b}\right)^{2} \stackrel{1}{t}_{Y^{E}(0)} \prod_{i=1}^{n} T_{i}^{E}+\left(\frac{m \pi}{a}\right)^{2} \stackrel{1}{t}^{M}(0)}{\left(\frac{m \pi}{a}\right)^{2}+\left(\frac{n \pi}{b}\right)^{2} T_{i}^{M}} \cos \frac{m \pi x^{\prime}}{a} \cos \frac{m \pi x}{a} \sin \frac{n \pi y^{\prime}}{b} \sin \frac{n \pi y}{b} .
$$

Let the dipole is at the source point $x^{\prime}=a / 2, z^{\prime}=0$ and has the length $L$. If the point of view is at $x=a / 2$ and $z=z_{n}$, the $E_{y}$ field component is described as follows:

$$
E_{y}=\sum_{m=0}^{\infty} \sum_{n=0}^{\infty} \frac{1}{a b} \frac{1}{\left(\frac{m \pi}{a}\right)^{2}+\left(\frac{n \pi}{b}\right)^{2}}\left[\left(\frac{n \pi}{b}\right)^{2} \frac{\varepsilon_{m} \varepsilon_{n}}{k^{2}} \frac{\prod_{\dot{x}^{=1}}^{n} T_{i}^{E}}{Y^{E}(0)}+\left(\frac{m \pi}{a}\right)^{2} \frac{\prod_{\dot{j}^{=}}^{n} T_{i}^{M}}{Y^{M}(0)}\right] \frac{b}{\pi n}\left(\cos \frac{n \pi L}{b}-1\right) \cos ^{2} \frac{m \pi}{2} \sin \frac{n \pi y}{b} .
$$

Unlike the structures considered in [13], waveguide structures have dispersion. There are frequencies, for which the considered waveguides with periodically changing characteristics have only evanescent modes. This frequency range for some electromagnetic problems is also of interest, for example, in the design of attenuators.

It is known that the undisturbed periodic structure strongly blocks the propagation of electromagnetic waves and acts as a notch filter. Electromagnetic waves bounce off this structure. Let us consider the 25-layer section of the rectangular waveguide of 23 by $10 \mathrm{~mm}$ cross-section with periodical changing dielectric layers (odd layers thickness $7.1 \mathrm{~mm}, \varepsilon=1.0$; even layers thickness $3.9 \mathrm{~mm}, \varepsilon=2.63$, number of odd layers 13 , number of even layers 12). Fig. 2 shows the frequency dependencies of the transmission $S_{21}$ and reflection $S_{11}$ coefficients. The stopband at the level of $-30 \mathrm{~dB}$ is $3.31 \mathrm{GHz}$ wide.

The presence of any inhomogeneity in the form of a layer of the same thickness, but with different electrical characteristics or with the same characteristics, but of a different thickness, causes the propagation of electromagnetic waves with small reflection in a narrow frequency band. The photonic structures with defects have narrow passbands and wide stopbands. Let the 12-th layer have other options. First, change the thickness of this layer. If the thickness of the layer reduces from 3.9 to $1.0 \mathrm{~mm}$, there is a narrow passband at the frequency $11.56 \mathrm{GHz}$. At zero layer thickness, the peak is shifted to the frequency $12.46 \mathrm{GHz}$, i.e., by $900 \mathrm{MHz}$ with a change in the thickness of $1 \mathrm{~mm}$, which allows, (e.g. for sufficiently high accuracy) to measure the thickness of the layer in experimental studies.
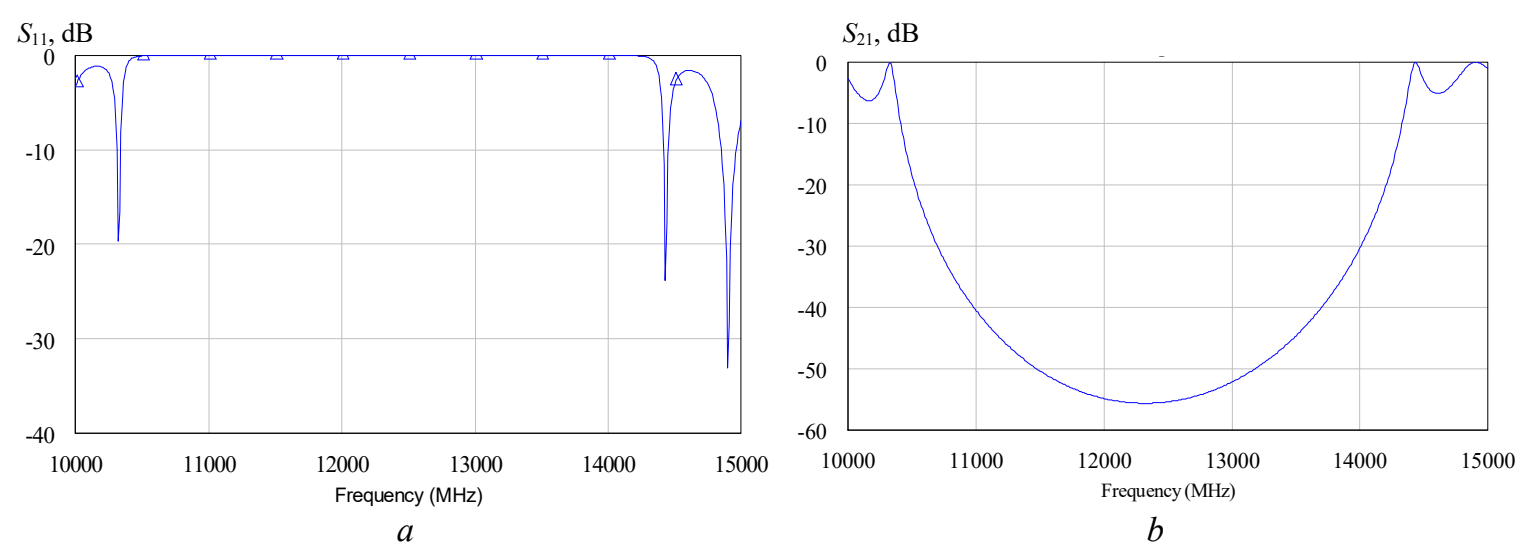

FIGURE 2. Reflection (a) and transmission (b) coefficients of the regular structure of a photonic crystal.

Now consider changing the permittivity, leaving the thickness constant. Peak of the passband shifts from 11.43 to $11.28 \mathrm{GHz}$ with the change in the layer permittivity from 1 to 1.12 . For the higher permittivity, a change in the 
passband peak frequency is more noticeable (change in the permittivity from 7.0 to 14.5 leads to peak shift from 13.48 to $11.07 \mathrm{GHz}$ ).

\section{CONCLUSION}

According to the obtained results using a vector network analyzer, one can determine the electrical parameters of the test layer with sufficiently high accuracy. Application of the Green's functions to analysis of the photonic crystals in a Cartesian coordinate system is discussed. In the case of analysis of the radially inhomogeneous structures, the representation of the Green's function in a cylindrical coordinate system can be recommended, as in [14].

The presented method for calculating the transmission coefficients remains correct in the terahertz and optical frequency ranges due to strict analytical expressions. It is noted that the solution of the inverse problem makes it possible to determine the thickness and electrical characteristics of the disturbed layer. The high sensitivity of the resonant frequency of the structure passband under the presence of a disturbed layer makes it possible to determine the electrodynamic characteristics of the studied materials with high accuracy.

\section{ACKNOWLEDGMENT}

This work was supported by the Grant of the Ministry of Science and Higher Education of the Russian Federation (project no 8.2538.2017/4.6).

\section{REFERENCES}

1. K. Sakoda, Optical Properties of Photonic Crystals, 2nd ed. Berlin Heidelberg: Springer-Verlag, 2005.

2. C. López, "Materials Aspects of Photonic Crystals," Advanced Materials, vol. 15, no. 20, pp. 1679-1704, 2003.

3. E. Yablonovitch, "Inhibited Spontaneous Emission in Solid-State Physics and Electronics," Phys. Rev. Lett., vol. 58, no. 20, pp. 2059-2062, 1987.

4. C. A. Kyriazidou, H. F. Contopanagos, and N. G. Alexopoulos, "Monolithic waveguide filters using printed photonic-bandgap materials," IEEE Transactions on Microwave Theory and Techniques, vol. 49, no. 2, pp. 297-307, Feb. 2001.

5. S. Li, H. Liu, Q. Sun, and N. Huang, "A Tunable Terahertz Photonic Crystal Narrow-Band Filter," IEEE Photonics Technology Letters, vol. 27, no. 7, pp. 752-754, Apr. 2015.

6. G. Shen et al., "A tunable electro-optic microwave photonic filter based on photonic crystal for $60 \mathrm{GHz}$ radio over fiber system," in 2013 12th International Conference on Optical Communications and Networks (ICOCN), 2013, pp. 1-4.

7. Y. Zhang et al., "Slow-Light Dispersion in One-Dimensional Photonic Crystal Racetrack Ring Resonator," IEEE Photonics Technology Letters, vol. 27, no. 10, pp. 1120-1123, May 2015.

8. M. N. Nawi, N. D. Rashid, D. D. Berhanuddin, B. Y. Majlis, M. A. Mahdi, and A. R. M. Zain, "Photonic crystal embedded waveguide for compact C-band band-pass filter," in 2018 IEEE International Conference on Semiconductor Electronics (ICSE), 2018, pp. 180-183.

9. M. Bellingeri, D. Cassi, L. Criante, and F. Scotognella, "Light Transmission Properties and Shannon Index in One-Dimensional Photonic Media With Disorder Introduced by Permuting the Refractive Index Layers," IEEE Photonics Journal, vol. 5, no. 6, pp. 2202811, Dec. 2013.

10. A. M. Vyunishev, P. S. Pankin, S. E. Svyakhovskiy, I. V. Timofeev, and S. Ya. Vetrov, "Quasiperiodic onedimensional photonic crystals with adjustable multiple photonic bandgaps," Opt. Lett., vol. 42, no. 18, p. 3602, Sep. 2017.

11. D. G. Dudley, "The Green's Function Method," in Mathematical Foundations for Electromagnetic Theory, IEEE, 1994.

12. A. Karpov, S. Knyazev, L. Lesnaya, and S. Shabunin, "Sandwich spherical and geodesic antenna radomes analysis," in 2016 10th European Conference on Antennas and Propagation (EuCAP), 2016, pp. 1-5.

13. L. B. Felsen and N. Marcuvitz, Radiation and Scattering of Waves. John Wiley \& Sons, 1994.

14. S. Daylis, S. Shabunin, "Green's Functions of Multi-layered Cylindrical Structures for Electromagnetic Radiation, Propagation and Scattering Problems Solution," in Ural Radio Engineering Journal, vol. 1, no. 1, pp. 91-119, 2017. 